\title{
THE INTEGRABILITY OF A SEQUENCE OF FUNCTIONS*
}

\author{
BY \\ R. L. JEFFERY
}

1. Introduction. Let $f=f_{1}, f_{2}, \cdots$ be a sequence of functions summable on the measurable set $E$, and convergent on $E$ to the summable function $F$. If

$$
\lim _{n \rightarrow \infty} \int_{E} f_{n} d x=\int_{E} F d x
$$

the sequence is said to be integrable. If the above equality holds when the set $E$ is replaced by any measurable part of $E$ the sequence is said to be completely integrable. These questions of integrability and complete integrability have received considerable attention from various writers. $\dagger$ It has been shown by Vitali that the equi-convergence $\$$ of the sequence of integrals is both necessary and sufficient for complete integrability. It would then follow that this condition is sufficient for integrability. One can, however, easily construct examples which show that it is not necessary. The chief aim of the present paper is to determine conditions which are both necessary and sufficient for the integrability of the sequence $f$. This is accomplished by methods which yield, as special cases, some of the results already obtained by Vitali and de la Vallée Poussin.

2. Definitions and preliminary results. In what follows, without again making mention of it, we shall use $e$ to denote any measurable sub-set of $E$. We further define $S(l, \eta), \eta>0$ and arbitrary, to be the part of $E$ for which $\left|F-f_{n}\right|<\eta(n \geqq l)$, and $C(l, \eta)$ its complement on $E ; e(l, n)_{+}$and $e(l, n)_{-}$the parts of $C(l, \eta)$ for which $f_{n} \geqq 0, f_{n}<0$ respectively. It is easily verified that these sets are measurable, and that $m C(l, \eta)$ tends to zero as $l$ becomes infinite. Finally we use $g=g_{1}, g_{2}, \cdots$ to denote a sub-sequence $f_{n_{1}}, f_{n_{2}}, \cdots$ of $f$.

We shall have occasion to use

* Presented to the Society, December 27, 1929; received by the editors April 26, 1930.

† Osgood, American Journal of Mathematics, vol. 19, p. 182; Hobson, Proceedings of the London Mathematical Society, (1), vol. 35, p. 254; W. H. Young, Proceedings of the London Mathematical Society, (2), vol. 1, p. 89; Arzelà, Memorie, Reale Accademia delle Scienze, Bologna, (5), vol. 8, p. 703; Vitali, Rendiconti del Circolo Matematico di Palermo, vol. 23, p. 137; de la Vallé Poussin, these Transactions, vol. 16, p. $444 \mathrm{ff}$.

$\ddagger$ Loc. cit.

§ Hobson, Real Variable, second edition, vol. II, p. 208. 
A. Corresponding to $\epsilon>0$ there exists $\delta>0$ such that if $m e<\delta$ then

$$
\left|\int_{e S(l, \eta)} f_{n} d x\right|<\epsilon \quad(n \geqq l) .
$$

This readily follows from the summability of $F$ and the fact that, on $S(l, \eta), f_{n}=F+t_{n}$, where $\left|t_{n}\right|<\eta$ for $n \geqq l$.

We now prove

I. It is necessary and sufficient for the integrability of $f$ that for every $\eta>0$

$$
\lim _{l \rightarrow \infty} \int_{C(l, \eta)} f_{n} d x=0 \quad(n \geqq l) .
$$

To show that this is sufficient we write

$$
\left|\int_{E} f_{n} d x-\int_{E} F d x\right| \leqq \int_{S(l, \eta)}\left|f_{n}-F\right| d x+\int_{C_{(l, \eta)}}\left|f_{n}-F\right| d x .
$$

We thus see that for $l$ sufficiently large and for $\eta$ sufficiently small the lefthand side of this inequality is arbitrarily small for all $n \geqq l$.

The condition is also necessary. Obviously

$$
\int_{C(l, \eta)} f_{n} d x=\int_{C(l, \eta)} F d x+\int_{E}\left(f_{n}-F\right) d x-\int_{S(l, \eta)}\left(f_{n}-F\right) d x .
$$

Then for any $\eta$ and $n \geqq l$, we have

$$
\left|\int_{C(\boldsymbol{l}, \eta)} f_{n} d x\right| \leqq\left|\int_{C(\boldsymbol{l}, \eta)} F d x\right|+\left|\int_{E}\left(f_{n}-F\right) d x\right|+\eta \cdot m E .
$$

Now if $0<\eta^{\prime}<\eta$, then $C\left(l, \eta^{\prime}\right)$ contains $C(l, \eta)$ and $C\left(l, \eta^{\prime}\right)=C(l, \eta)+C\left(l, \eta^{\prime}\right)$ $\cdot S(l, \eta)$ so that

$$
\begin{aligned}
\left|\int_{C(l, \eta)} f_{n} d x\right| & \leqq\left|\int_{C\left(l, \eta^{\prime}\right)} f_{n} d x\right|+\left|\int_{C\left(l, \eta^{\prime}\right) S(l, \eta)} f_{n} d x\right| \\
& \leqq\left|\int_{C\left(l, \eta^{\prime}\right)} f_{n} d x\right|+\eta \cdot m C\left(l, \eta^{\prime}\right)+\int_{C\left(l, \eta^{\prime}\right)}|F| d x \\
& \leqq 2 \int_{C\left(l, \eta^{\prime}\right)}|F| d x+\left|\int_{E}\left(f_{n}-F\right) d x\right|+\eta^{\prime} \cdot m E+\eta \cdot m C\left(l, \eta^{\prime}\right),
\end{aligned}
$$

the last inequality following from (1) by replacing $\eta$ by $\eta^{\prime}$. The necessity of the condition follows at once from this inequality, since it is valid for all $\eta^{\prime}<\eta$ and since $m C\left(l, \eta^{\prime}\right)$ tends to zero as $l$ becomes infinite.

3. The integral of $f_{n}$ over $e$ bounded in $e$ and $n$. We first prove 
B. If the sequence of functions $f=f_{1}, f_{2}, \cdots$ is summable on the measurable set $E$, converges to the function $F$ which is measurable on $E$, and is such that the integral of $f_{n}$ over $e$ is bounded in e and $n$, then $F$ is summable on $E$.

Let $M$ be the least upper bound of

$$
\int_{e}\left|f_{n}\right| d x
$$

for all $e$ of $E$ and all $n$. Further let $E_{N}$ be the part of $E$ for which

$$
-N \leqq F \leqq N \text {. }
$$

Then $F$ will be summable on $E$ if

$$
\int_{\boldsymbol{E}_{N}}|F| d x
$$

is bounded as to $N$. Now

$$
\begin{aligned}
\int_{E_{N}}|F| d x & =\int_{E_{N} S(l, \eta)}|F| d x+\int_{E_{N} C(l, \eta)}|F| d x \\
& \leqq \int_{E_{N} S(l, \eta)}\left(\left|F-f_{n}\right|+\left|f_{n}\right|\right) d x+\int_{E_{N} C(l, \eta)}|F| d x \\
& \leqq \eta \cdot m E+M+N \cdot m C(l, \eta) .
\end{aligned}
$$

Since $m C(l, \eta)$ approaches zero as $l$ approaches infinity independently of $N$, we have at once the desired boundedness.

If the sequence of integrals is equi-convergent then, obviously, the integral of $f_{n}$ over $e$ is bounded in $e$ and $n$. We thus get

$\mathrm{B}^{\prime *}$. If the sequence of summable functions $f$ converges on $E$ to a measurable function $F$, and if the sequence of integrals is equi-convergent, then $F$ is summable.

If the sequence of integrals is not equi-convergent, by making use of $A$ it is easy to show that there exists $\lambda>0$ and a sequence of values $n_{1}, n_{2}, \cdots$ of $n$ such that either

$$
\int_{e\left(l_{i}, n_{i}\right)_{+}} f_{n_{i}} d x>\lambda \text { or } \int_{e\left(l_{i}, n_{i}\right)-} f_{n_{i}} d x<-\lambda \quad\left(l_{i}=n_{i}, i=1,2, \cdots\right) .
$$

Assuming that the first holds we can select from the sequence $n_{i}$ a subsequence $n_{j}$ such that for $\epsilon$ arbitrary

* de la Vallée Poussin, loc. cit., Theorem I. 


$$
\int_{e\left(l_{j}, n_{j}\right)_{+}} f_{n_{j}} d x>\lambda \text { and } \int_{C\left(l_{j+1}, \eta\right)}\left|f_{n_{j}}\right| d x<\epsilon .
$$

Set $e_{j}=\left\{C\left(l_{j}, \eta\right)-C\left(l_{j+1}, \eta\right)\right\} e\left(l_{j}, n_{j}\right)_{+}$. Then

$$
\int_{e_{j}} f_{n_{j}} d x>\lambda-\epsilon \text {. }
$$

Also $e_{1}+e_{2}+\cdots+e_{j-1}$ is contained in $S\left(l_{j}, \eta\right)$ and in $C\left(l_{1}, \eta\right)$, and $e_{j+1}+\cdots$ is contained in $C\left(l_{j+1}, \eta\right)$. Hence if $l_{1}$ is taken sufficiently large and $e=\sum e_{j}$, we have both

$$
\int_{e}|F| d x<\epsilon, \quad \text { and } \int_{e} f_{n_{j}} d x>\lambda-2 \epsilon \quad(j=1,2, \cdots)
$$

which shows that the sequence is not completely integrable. In case $f$ is such that the sequence of integrals is equi-convergent, then, if $E^{\prime}$ is any measurable part of $E$, complete integrability readily follows from

$$
\int_{E^{\prime}}\left|F-f_{n}\right| d x=\int_{E^{\prime} S(l, \eta)}\left|F-f_{n}\right| d x+\int_{E^{\prime} C(l, \eta)}\left|F-f_{n}\right| d x \quad(n \geqq l) .
$$

We thus have

$C^{*}$. If a sequence of summable functions converges on $E$ to a summable function $F$, it is then necessary and sufficient for complete integrability that the sequence of integrals be equi-convergent.

Let $g=g_{1}, g_{2}, \ldots$ be any sub-sequence of $f$, and $\delta$ any positive number. Let $U(g, n, \delta)$ and $L(g, n, \delta)$ be the least upper bound and greatest lower bound respectively of

$$
\int_{e} g_{i} d x \quad(i=1,2, \ldots ; n)
$$

for every $e$ with $m e<\delta$. It is evident that, for a given $\delta, U(g, n, \delta)$ does not decrease as $n$ increases, and consequently, since the integral of $f_{n}$ over $e$ is bounded in $e$ and $n$, this function converges to a limit $U(g, \delta)$. But $U(g, \delta) \geqq 0$, and obviously does not increase as $\delta$ decreases. Hence $U(g, \delta)$ converges to a limit $U(g)$. Dealing in a similar manner with the function $L(g, n, \delta)$ we arrive at the corresponding limit $L(g)$. It is now possible to prove

II. If the sequence of summable functions $f$ converges on $E$ to a measurable function $F$, and if the integral of $f_{n}$ over $e$ is bounded in $n$ and $e$, it is then neces-

* See also de la Vallée Poussin, loc. cit., Theorem IV. 
sary and sufficient for the integrability of the sequence that $U(g)+L(g)=0$ for every $g$.

That $F$ is summable follows from B. To show that the conditions are necessary, let $f$ be integrable and assume that $U(g)+L(g) \neq 0$ for at least one g. For the sake of definiteness let

$$
U(g)+L(g)=\lambda>0 .
$$

Making use of $\mathrm{A}$ it is possible to fix $\delta$ so that both

$$
U(g, \delta)+L(g, \delta)>\frac{\lambda}{2}
$$

and if $m e<\delta$,

$$
\left|\int_{e S(l, \eta)} g_{n} d x\right|<\frac{\lambda}{4} \quad(n \geqq l) .
$$

Again, since the sequence is integrable, we can use I to fix $l=l^{\prime}$ for which both $m C(l, \eta)<\delta\left(l \geqq l^{\prime}\right)$, and

$$
\left|\int_{C(l, \eta)} g_{n} d x\right|<\frac{\lambda}{4} \quad\left(n \geqq l \geqq l^{\prime}\right) .
$$

But for any $l \geqq l^{\prime}$ it is evident that there exist $e$ and $n \geqq l$ such that we have

$$
\begin{aligned}
\int_{e(l, n)_{+}} g_{n} d x \leqq U(g, \delta) & \leqq \int_{e} g_{n} d x+\frac{\lambda}{8} \\
& \leqq \int_{e e(l, n)_{+}} g_{n} d x+\int_{e e(l, n)_{-}} g_{n} d x+\int_{e S(l, \eta)} g_{n} d x+\frac{\lambda}{8} \\
& \leqq \int_{e(l, n)_{-}} g_{n} d x+\frac{\lambda}{4} \quad\left(n \geqq l \geqq l^{\prime}\right) .
\end{aligned}
$$

Similarly we arrive at

$$
\int_{e(l, n)_{-}} g_{n} d x-\frac{\lambda}{4} \leqq L(g, \delta) \leqq \int_{e(l, n)_{-}} g_{n} d x \quad\left(n \geqq l \geqq l^{\prime}\right) .
$$

But (5) and (6) combine to contradict (4). We conclude, therefore, that the conditions of (2) are necessary.

The conditions are also sufficient. Suppose that, for every $g, U(g)+L(g)$ $=0$. If $f$ is not integrable then $\mathrm{I}$ is denied, and we can assume the existence of a number $\lambda>0$ and two sequences $l_{1}, l_{2}, \cdots$ and $n_{1}, n_{2}, \cdots$ where $n_{i} \geqq l_{i}$ and such that either 


$$
\int_{C\left(l_{i}, \eta\right)} f_{n_{i}} d x>\lambda \quad(i=1,2, \cdots),
$$

or the left-hand side of (7) is less than $-\lambda$. For definiteness suppose that (7) holds. Let $g_{i}=f_{n_{i}}$. Fix $\delta$ so that we have

$$
U(g, \delta)+L(g, \delta)<\frac{\lambda}{2}
$$

and so that for $m e<\delta$ we also have

$$
\left|\int_{e S\left(l_{i}, \vartheta\right)} g_{i} d x\right|<\frac{\lambda}{4} .
$$

Fix $i=i^{\prime}$ such that for $i \geqq i^{\prime}, m C\left(l_{i}, \eta\right)<\delta$. Then, obviously, we have

$$
U(g, \delta) \geqq \int_{e\left(l_{i}, n_{i}\right)_{+}} g_{i} d x
$$

By methods similar to those used in obtaining (5) it is possible to show that

$$
L(g, \delta) \geqq \int_{e\left(l_{i}, n_{i}\right)-} g_{i} d x-\frac{\lambda}{2} \quad\left(i \geqq i^{\prime}\right) .
$$

But evidently (8), (10), and (11) can now be used to contradict (7). We conclude, therefore, that the conditions are sufficient.

If the sequence $f$ is such that $f_{n}$ is uniformly bounded below, then $L(g)=0$ for every $g$. Furthermore, if the sequence is integrable, it follows from II that for every $g, U(g)=0$. But if, for every $g, U(g)=L(g)=0$, it is evident that the sequence of integrals is equi-convergent. We thus get

III. If the sequence $f$ converges on $E$ to a measurable function $F$, and if $f_{n}$ is uniformly bounded below, or above, it is then necessary and sufficient for the integrability of $f$ that the sequence of integrals be equi-convergent. ${ }^{*}$

We have seen that the equi-convergence of the sequence of integrals is both necessary and sufficient for complete integrability. This, with III, gives

III'. If the sequence $f$ is integrable, and is such that $f_{n}$ is uniformly bounded below, or uniformly bounded above, then $f$ is completely integrable.

One might hope that the existence of the double limit $\underset{n \rightarrow \infty}{\delta \rightarrow 0}\{U(g, n, \delta)$ $+L(g, n, \delta)\}=0$ would prove to be both necessary and sufficient for integrability. On account of II, it is evidently sufficient. An example shows that it is not necessary.

\footnotetext{
* Cf. de la Vallée Poussin, loc. cit., p. 448.
} 
Let $e_{n}$ be a sequence of distinct measurable sets on $E$ with a single limit point not belonging to any set of the sequence, and such that $m e_{n}$ tends to zero monotonically. Let $e_{n}^{\prime}$ be a similar sequence distinct from $e_{n}$ with a single limit point not belonging to any set of either sequence, and with $m e_{n}^{\prime}=2 m e_{n}$. Let $\lambda$ be any positive real number, and let $f_{n}=\lambda / m e_{n}$ on $e_{n}$, $f_{n}=-\lambda /\left(2 m e_{n}\right)$ on $e_{n}^{\prime}$, and $f_{n}=0$ elsewhere on $E$. We see that $f_{n}$ converges to zero, and for each $n$ the integral of $f_{n}$ over $E$ is zero. But for every $\delta>0$ and every $n$ it is possible to find $\delta^{\prime}<\delta$ and $n^{\prime} \geqq n$ such that

$$
U\left(f, n^{\prime}, \delta^{\prime}\right)+L\left(f, n^{\prime}, \delta^{\prime}\right)=\frac{\lambda}{2} .
$$

Let $\delta$ and $n$ be given. Take $n^{\prime} \geqq n$ and such that $m e_{n^{\prime}}<\delta$, and fix $\delta^{\prime}=m e_{n^{\prime}}$. Then $U\left(f, n^{\prime}, \delta^{\prime}\right)=\lambda$, and $L\left(f, n^{\prime}, \delta^{\prime}\right)=-\delta^{\prime} \lambda / m e_{n^{\prime}}=-\lambda / 2$, and these give (1).

4. The integral of $f_{n}$ over $e$ not bounded in $n$ and $e$. We prove

IV. If the sequence of summable functions $f$ converges on $E$ to the summable function $F$, it is necessary and sufficient for the integrability of the sequence that corresponding to every $g$ of $f$ and every $\epsilon>0$ there is a number $\delta_{g e}>0$ such that for $\delta<\delta_{0 \ell}$ it is possible to find $n=n_{\delta}$ for which $|U(g, n, \delta)+L(g, n, \delta)|<\epsilon\left(n \geqq n_{\delta}\right)$.

Let $f$ be integrable. Suppose that for some $g$ the conditions of IV do not hold. If for this $g$ it so happens that for every $\delta$ only one of the functions $U(g, n, \delta), L(g, n, \delta)$ is unbounded in $n$, it is very easy to show that $\mathrm{I}$ is contradicted. If for every $\delta$ both these functions are bounded, then the methods of II can be used to contradict I. We thus have to consider only the case for which both these functions are, for every $\delta$, unbounded in $n$. In such a case there exists a number $\lambda>0$ and independent of $\delta$, and for each $\delta$ a sequence of values $n_{1}, n_{2}, \cdots$ of $n$ for which either

$$
U\left(g, n_{i}, \delta\right)+L\left(g, n_{i}, \delta\right)>\lambda \quad(i=1,2, \cdots),
$$

or the left-hand side of (1) is less than $-\lambda$. For the sake of definiteness let (1) hold. Since $\lambda$ is independent of $\delta$ we can consider only such values of $\delta$ that for $m e<\delta$ we have

$$
\left|\int_{e S(l, \eta)} g_{n} d x\right|<\frac{\lambda}{4} \quad(n \geqq l) .
$$

Since for any $\delta$ both the functions involved in (1) become monotonically infinite with $i$, it is possible to choose from $n_{i}$ a sub-sequence $n_{i}$ such that

$$
U\left(g, n_{j}, \delta\right)>U\left(g, n_{j-1}, \delta\right)+\frac{\lambda}{4}
$$


and

$$
L\left(g, n_{j}, \delta\right)<L\left(g, n_{j-1}, \delta\right)-\frac{\lambda}{4}
$$

both hold. It then follows from (3) that there exists some $n_{j}^{\prime}$ where $n_{j-1} \leqq n_{j}^{\prime}$ $\leqq n_{j}$, and some $e_{j}$ with $m e<\delta$, for which

$$
\int_{e_{j}} g_{n_{j}^{\prime}} d x>U\left(g, n_{j}, \delta\right)-\frac{\lambda}{4} .
$$

Then, by making use of (2) and the methods used in obtaining (5) and (6) in the proof of II, we arrive at

$$
\int_{e\left(l, n^{\prime}\right)+} g_{n j}^{\prime} d x>U\left(g, n_{j}, \delta\right)-\frac{\lambda}{2} .
$$

But for $j$ sufficiently large, $m e\left(l, n_{j}^{\prime}\right)_{-}<\delta\left(l=n_{j}^{\prime}\right)$, and then we evidently have

$$
\int_{e\left(l, n j^{\prime}\right)-} g_{n j}^{\prime} d x \geqq L\left(g, n_{j}, \delta\right) .
$$

But (5), (6), and (1) can now be used to contradict I. We conclude, therefore, that the conditions of IV are necessary.

In order to show that the conditions are sufficient, let IV hold and suppose that $f$ is not integrable. Then $I$ is denied. Consequently, there exists a number $\lambda>0$ and two sequences $l_{1}, l_{2}, \cdots$ and $n_{1}, n_{2}, \cdots$ where $n_{i} \geqq l_{i}$ and such that either

$$
\int_{C\left(l_{i}, \eta\right)} f_{n_{i}} d x>\lambda \quad(i=1,2, \cdots)
$$

holds, or the left side of (1) is less than $-\lambda$. For the sake of definiteness assume that (1) holds, and set $g_{i}=f_{n_{i}}$. It is then possible, by methods similar to those we have used above, to show that the hypotheses of IV are contradicted. This leads to the conclusion that the conditions of IV are sufficient.

The example given above can easily be modified to show that when the integral of $f_{n}$ over $e$ is unbounded the double limit $\underset{n \rightarrow \infty}{\delta \rightarrow 0}\{U(g, n, \delta)+L(g, n, \delta)\}$ $=0$ is not necessary for integrability.

Acadia University, Wolfville, N. S. 